\title{
Insect Fauna associated with the Tea Ecosystem of North Bengal, India
}

\author{
Bulganin Mitra*, Suresh Kumar Shah and Purnendu Mishra \\ Zoological Survey of India, H.Q. Office, M- Block, New Alipore, Kolkata - 700053, \\ West Bengal, India; bulganinmitra@gmail.com
}

\begin{abstract}
Present communication reports 167 species belonging to 139 genera of 42 families under 6 orders of insects from the tea gardens of North Bengal. Of them, Lepidoptera shares maximum number of species (77), followed by Hemiptera (29), Diptera (24), Coleoptera (19), Odonata (12) and Orthoptera (06). Among them, 20 species of Lepidoptera, 05 species of Hemiptera, 05 species of Coeloptera and 03 species of Orthoptera are found as tea pests. Besides this, 01 species of Diptera and Odonata are found as predators of tea pest. In addition to this, 14 species of Hemiptera, 11 species of Coleoptera and 09 species of Lepidoptera reported as pests/borers of other crops and timber plants. In addition to this, 03 species of Hemiptera and 03 species of Diptera as predator and 16 species of Diptera as flower visitors/pollinators in other ecosystem are also found.
\end{abstract}

Keywords: North Bengal, Pest, Pollinator, Predator, Tea Garden

\section{Introduction}

Insects are among the major animal visitors of any crop fields and act as pests, pollinators or some other biologically important organism to a particular plant including tea gardens. Therefore, accurate identification of insects at genus and species level is essential for understanding their biological role in a particular ecosystem.

Tea (Camellia sinensis (L.) O. Kuntze) is a popular beverage all over the world. Globally, 1031 arthropod species are associated with tea; a small number of pests (about 3\%) are common throughout the world (Hazarika et al., 2009). All parts of the plant, leaf, stem, root, flower, and seed, are fed upon by at least one pest species, resulting in 11\%-55\% loss in yield if left unchecked (Hazarika et al., 2009). Tea with perennial foliage is infested by about 167 insect species in the North-Eastern tea growing regions of India (Das et al., 2010) including the Dooars and Terai. Besides the role of insects in tea ecosystem as pest, they are serving other functional role as predator, parasites and pollinator. Bezbaruah, (1975) stated that, insect pollinators contribute to pollination success in tea.
Majority of the works in North Bengal tea gardens are restricted with insect pest, predator, and parasites and their control. Khewa and Mukhopadhyay (2010) worked on biopesticides of Arctonis submarginata. Roy et al. (2010) evaluated the effectiveness of acaricides/insecticides in killing the eggs of Helopeltis theivora Waterhouse and Oligonychus coffeae Nietner in North Bengal. Roy et al. (2010) studied the bio efficacy of coccinellid predators on major tea pests. Das et al. (2010) studied the seasonal occurrence of the three lepidopteron species from Terai tea plantations. Das et al. (2010) reported 94 species of predators and 33 of parasitoids as natural enemies of arthropods in sub-Himalayan tea plantations of North Bengal, India. After that, Mitra et al. (2014a and 2014b) published a list of 07 species of Orthoptera and 28 species of Hemiptera from tea plantations of Dooars. Biswas et al. (2015) reported 14 species of moths (Lepidoptera: Erebidae) associated with the tea plantations of northern West Bengal. Shah and Mitra (2015) also published a list of 39 species as tea associated moths, of which, 15 species were recognized as serious pests of tea. Documentation of butterflies has also been done by Mitra et al. (2015),

\section{* Author for correspondence}


where 37 species were observed to visit in tea plantations. Apart from this, the interactions of 12 species of Odonata (Shah et al., 2015) and 13 species of Coleoptera (Mitra et al., 2016) have also been recorded from this ecosystem. Recently, Mitra et al. (2017) documented 24 species belonging to 08 families of dipteran flies and their association with the tea plants.

Apart from these, there are many more insect species unknown to us in tea gardens. They may be beneficial or harmful to the tea plants. Without regular surveys and proper identification of these species, it is not possible to undertake any action on them.

Field surveys were conducted in 74 tea gardens and its adjoining areas from 04 districts i.e., Jalpaiguri, Alipurduar, Kochbihar and Darjeeling of northern West Bengal from 2012-2016.

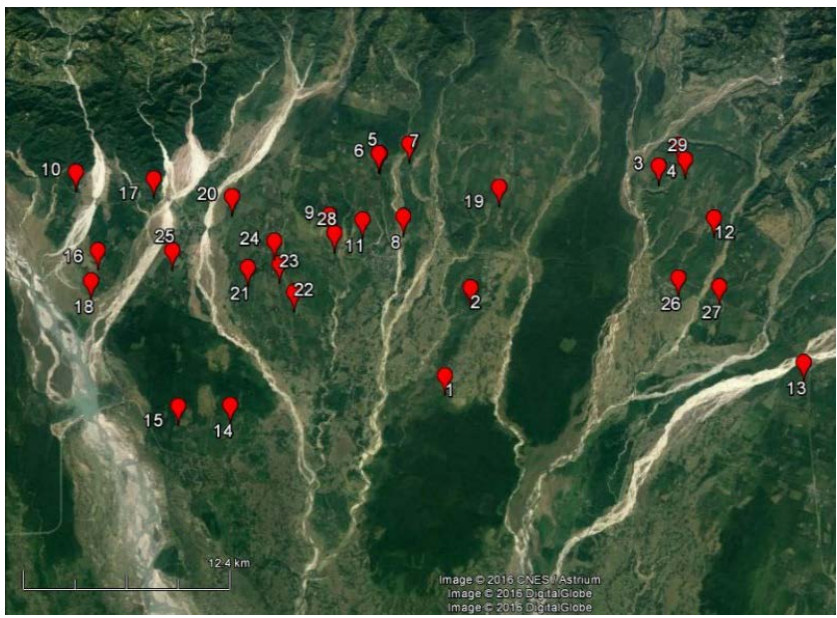

Figure 1. Tea gardens of Jalpaiguri district (Sr. No. 1-29).

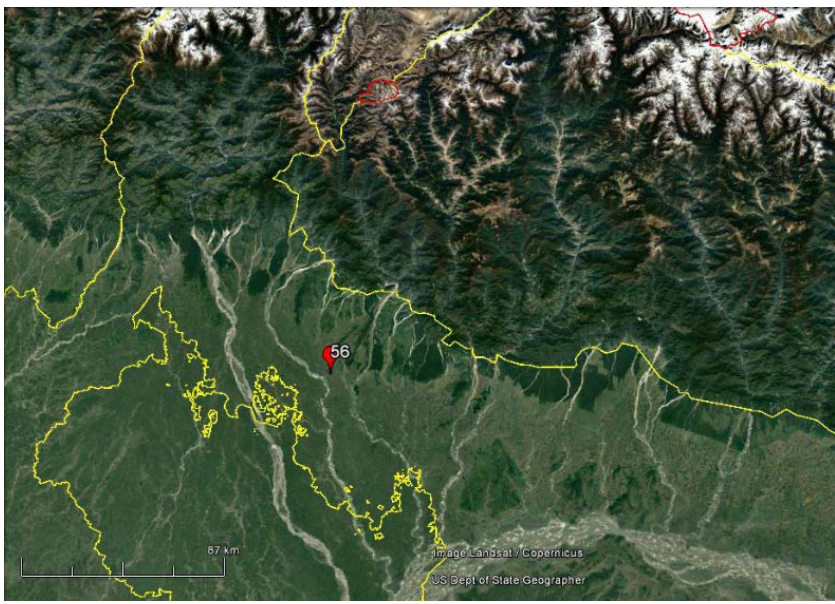

Figure 3. Tea garden of Kochbihar district (Sr. No. 56).
167 species belonging to 139 genera of 42 families under 6 orders of insects are reported from the tea gardens of North Bengal. Of them, Lepidoptera (77 species), followed by Hemiptera (29 species), Diptera (24 species), Coleoptera (19 species), Odonata (12 species) and Orthoptera (06 species) were collected and identified

\section{Material and Methods}

\section{Study area}

Survey was conducted in Dooars and the hilly area of Darjeeling of West Bengal from 2012 to 2016 in three districts of North Bengal and visited 74 tea gardens (Map.1-4). The serial number (1-74) has been maintained as per Table 1.

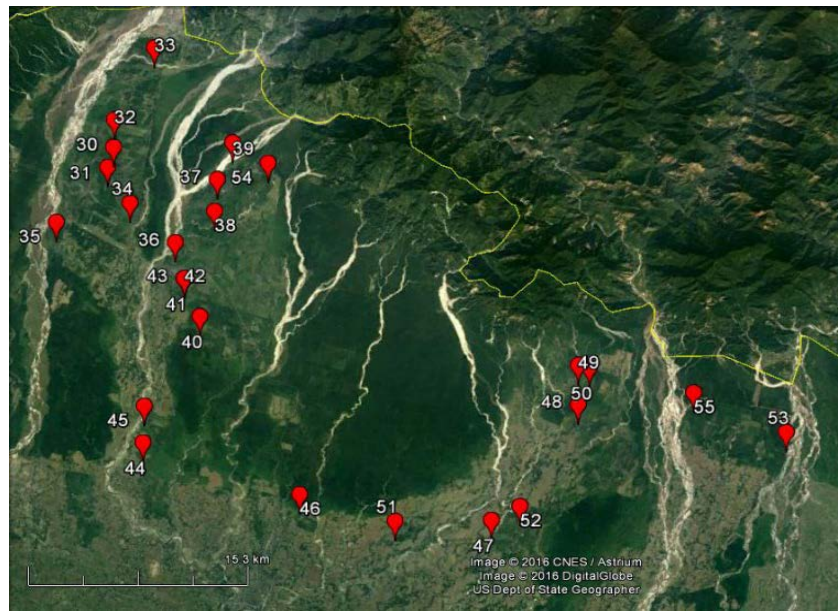

Figure 2. Tea gardens of Alipurduar district (Sr. No. 30-55).

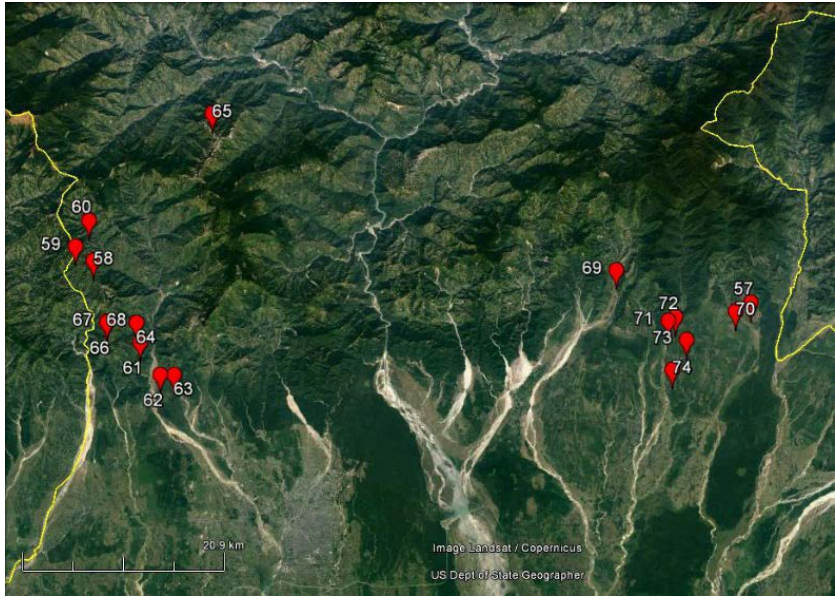

Figure 4. Tea gardens of Darjeeling district (Sr. No. 57-74). 


\section{Methodology}

The insects were randomly collected from various types of habitats observed in tea ecosystem. At day time, collections were operated by sweeping insect net, beating vegetation and handpicking method. The nocturnal insects were collected between 5.30 p.m. to 10.00 p.m. by using light trap (a white screen and a 27 Watt CFL lamp operated by portable rechargeable UPS machines). Collection permission was not granted after $10 \mathrm{PM}$., due to elephants and leopards movement. Butterflies and other macro insects were mostly photographed and identified in the field itself. The doubtful specimens were collected, killed by putting them in killing bottle containing ethyl acetate and brought to the laboratory in insect envelops. Soft bodied insects like Hymenopterans and some dipterans etc. were collected and preserved in glass vials containing 90 percent ethyl alcohol. The collected specimens, after bringing in Z.S.I. laboratory, were further processed using standard methods (Arora, 1986). The collected specimens

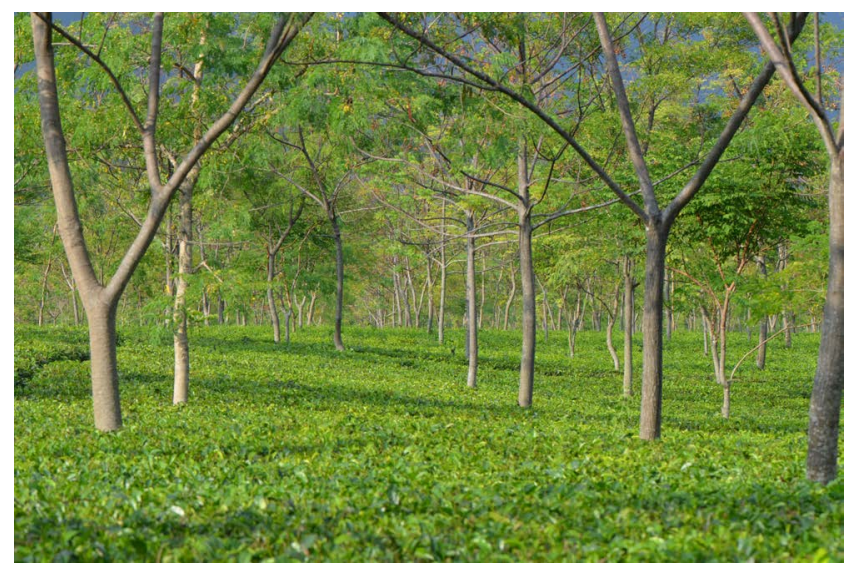

Figure 5. Tea garden before pruning.

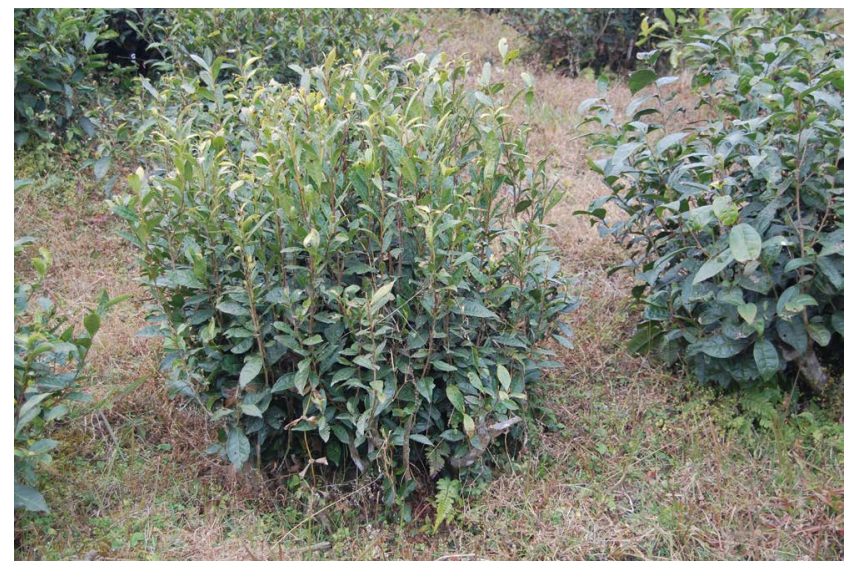

Figure 7. Tea plant. were identified after setting and pinning by Scientists of Z.S.I., Kolkata.

\section{Brief Description of the Plant}

C. sinensis (Linnaeus) belonging to the family Theaceae is an evergreen tree or shrub that attains a height of $10-15 \mathrm{~m}$ in the wild and $0.6-1.5 \mathrm{~m}$ when cultivated. These are characterized by the presence of light green leaves, short stalked, coriaceous, alternate, lanceolate, serrate margin, glabrous or pubescent beneath, varying in length from $5-30 \mathrm{~cm}$ and about $4 \mathrm{~cm}$ width. Mature leaves are bright green colored, smooth and leathery while young leaves are pubescent. Flowers are white fragrant, $2.5-4 \mathrm{~cm}$ in diameter, found in solitary or in clusters of two or four. Flowers bear numerous stamens with yellow anther and produce brownish red capsules (Ross, 2005). Fruit is a flattened, smooth, rounded trigonous three celled capsule, seed solitary in each, size of a small nut (Biswas, 2006).

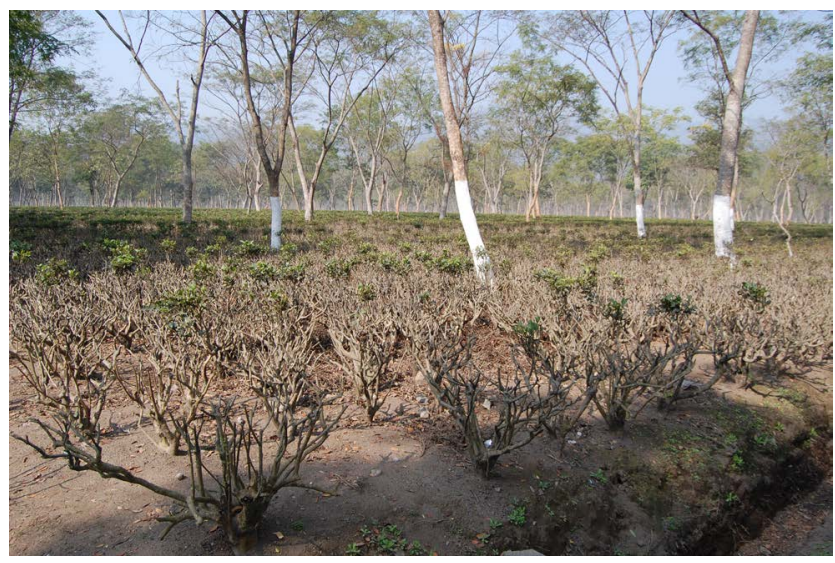

Figure 6. Tea garden after pruning.

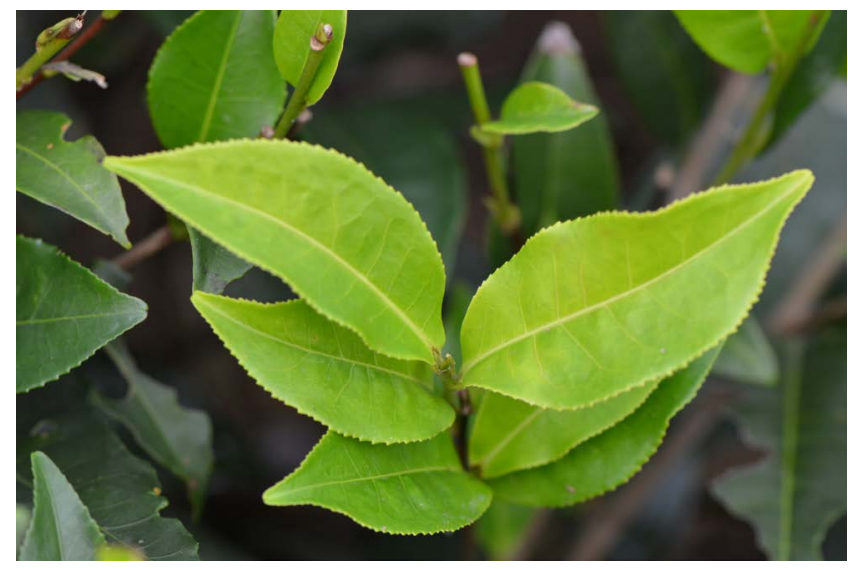

Figure 8. Tea leaves. 


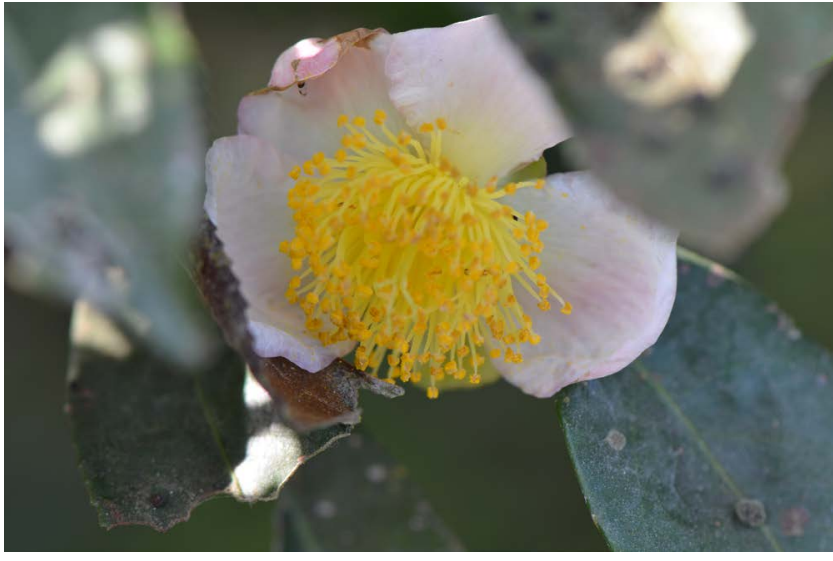

Figure 9. Tea flower.

\section{Observations}

74 tea gardens in 4 districts of North Bengal were surveyed, within the range of $26^{\circ} 29.526^{\circ}$ to $27^{\circ} 03.070^{\circ}$ North latitude to $88^{\circ} 08.916^{\circ}$ to $89^{\circ} 43.517^{\circ}$ East longitude and altitude from $127^{\prime}$ to $6630^{\prime}$ (Table 1). Of them, 29 tea gardens are in the Jalapiguri, 26 tea gardens

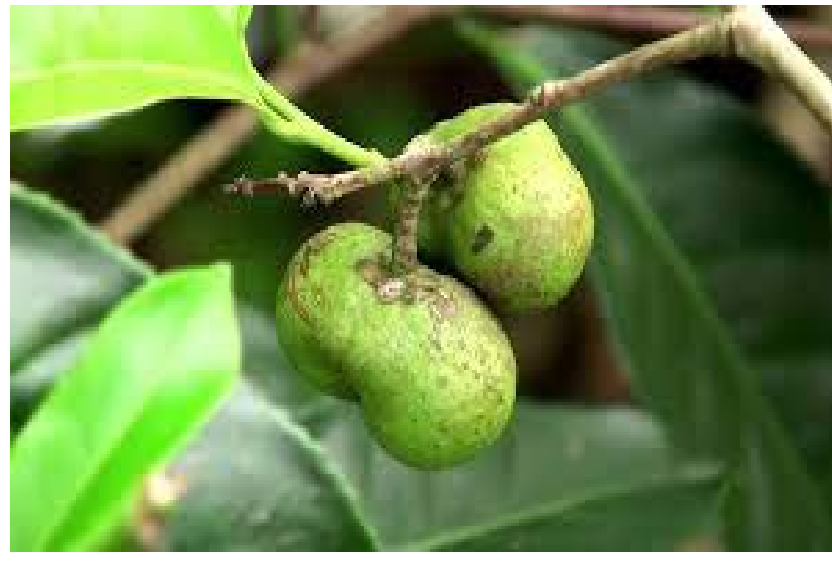

Figure 10. Tea fruits.

in Alipurduar, 18 in Darjeeling and single in Kochbihar district. Most of the tea gardens were visited once in a year but few gardens visited for more than one time (Table 1). In all, 167 species belonging to 139 genera of 41 families under 6 order of insects were reported and identified from the tea gardens (74) of North Bengal.

Table 1. Details of visited Tea gardens of North Bengal

\begin{tabular}{|c|c|c|c|c|c|}
\hline Sr No. & Name of the Tea gardens & District & Geographical Position & $\begin{array}{l}\text { Alt. (ft. } \\
\text { ASL) }\end{array}$ & Visited months \\
\hline 1 & BARODIGHI & Jalpaiguri & N26 48.193", E $088^{\circ} 47.790^{\prime \prime}$ & 470 & Jan-Feb \\
\hline 2 & BATABARI & Jalpaiguri & N26 51.149", E $088^{\circ} 48.045^{\prime \prime}$ & 570 & December \\
\hline 3 & HILLA & Jalpaiguri & $\begin{array}{l}\text { N26 56.244", E } 088^{\circ} 53.807^{\prime \prime} \\
\text { N } 2656.847, \text { E } 8853.509 \\
\text { N26 57.039", E } 088^{\circ} 54.370^{\prime \prime}\end{array}$ & $\begin{array}{l}1138 \\
950 \\
1158 \\
\end{array}$ & $\begin{array}{l}\text { Jan-Feb, July, December, } \\
\text { June, August, January }\end{array}$ \\
\hline 4 & NAGRAKATA TRA & Jalpaiguri & N26 57.039", E $088^{\circ} 54.370^{\prime \prime}$ & 1158 & Jan-Feb, July \\
\hline 5 & MEENGLAS & Jalpaiguri & N26 $54.777^{\prime \prime}$, E $088^{\circ} 43.834^{\prime \prime}$ & 667 & $\begin{array}{l}\text { Jan-Feb, December, } \\
\text { November }\end{array}$ \\
\hline 6 & MAL NADY & Jalpaiguri & $\begin{array}{l}\mathrm{N} 26^{\circ} 54.776^{\prime \prime}, \text { E } 88^{\circ} 43.785^{\prime \prime}, \mathrm{N} 26^{\circ} \\
54.776^{\prime \prime}, \text { E } 88^{\circ} 43.785^{\prime \prime}\end{array}$ & 873 & December \\
\hline 7 & NAKHATI & Jalpaiguri & N26 55.289", E $088^{\circ} 44.802^{\prime \prime}$ & 882 & December, January \\
\hline 8 & SOONGACHI & Jalpaiguri & N26 $52.952^{\prime \prime}$, E $088^{\circ} 45.126^{\prime \prime}$ & 608 & $\begin{array}{l}\text { December, August, } \\
\text { January }\end{array}$ \\
\hline 9 & RANGAMUTTEE & Jalpaiguri & N26 52.497", E $088^{\circ} 42.489^{\prime \prime}$ & 575 & December, August \\
\hline 10 & WASABARI & Jalpaiguri & N26 $52.156^{\prime \prime}$, E $088^{\circ} 33.151^{\prime \prime}$ & 570 & Jan-Feb \\
\hline 11 & NEDAM & Jalpaiguri & N26 52.577", E $088^{\circ} 43.695^{\prime \prime}$ & 600 & July \\
\hline 12 & KURTI & Jalpaiguri & N26 54.975", E $088^{\circ} 56.161^{\prime \prime}$ & 907 & July, November \\
\hline 13 & RED BANK & Jalpaiguri & 26 51.003', E 089 00.432', & 644 & July \\
\hline 14 & ANANDPUR & Jalpaiguri & $\begin{array}{l}\text { N26 } 45.814^{\prime \prime}, \text { E } 088^{\circ} 40.385^{\prime \prime}, \text { N26 } \\
45.370, \text { E } 08839.658\end{array}$ & $\begin{array}{l}409 \\
415\end{array}$ & December, June \\
\hline
\end{tabular}




\begin{tabular}{|c|c|c|c|c|c|}
\hline 15 & KAILASHPUR & Jalpaiguri & $\begin{array}{l}\text { N26 } 45.432^{\prime \prime}, \text { E } 088^{\circ} 38.545^{\prime \prime} \\
\text { N } 2645.370, \text { E } 08839.658\end{array}$ & $\begin{array}{l}442 \\
415\end{array}$ & December, June \\
\hline 16 & SONALI & Jalpaiguri & N26 $49.823^{\prime \prime}$, E $088^{\circ} 34.511^{\prime \prime}$ & 465 & Jan-Feb, November \\
\hline 17 & BAGRACOTE & Jalpaiguri & N26 52.471", E $088^{\circ} 35.969^{\prime \prime}$ & 551 & $\begin{array}{l}\text { November, August, } \\
\text { January }\end{array}$ \\
\hline 18 & LEESH RIVER & Jalpaiguri & $\mathrm{N} 26^{\circ} 48.813^{\prime}, \mathrm{E} 088^{\circ} 34.495^{\prime \prime}$ & 495 & November \\
\hline 19 & KILLCOTT & Jalpaiguri & N26 $54.520^{\prime \prime}$, E $088^{\circ} 48.304^{\prime \prime}$ & 876 & December \\
\hline 20 & RANICHHERA & Jalpaiguri & N26 $52.435^{\prime \prime}$, E $088^{\circ} 38.888^{\prime \prime}$ & 565 & June \\
\hline 21 & DAMDIM TG & Jalpaiguri & N 26 50.290, E O88 39.982" & 474 & June \\
\hline 22 & BETGURI TG & Jalpaiguri & N 26 49.817, E O88 41.806" & 497 & June \\
\hline 23 & KUMLAI TG & Jalpaiguri & N 26 50.626, E O88 41.070" & 533 & June \\
\hline 24 & DIANA TG & Jalpaiguri & N 26 51.308, E O88 40.723" & 533 & June \\
\hline 25 & ODLABARI & Jalpaiguri & N26 $50.323^{\prime \prime}$, E $088^{\circ} 37.168^{\prime \prime}$ & 456 & December \\
\hline 26 & BHOGOTPUR & Jalpaiguri & $\mathrm{N} 26^{\circ} 52.848$, E $088^{\circ} 55.350^{\prime \prime}$ & 587 & June \\
\hline 27 & GRASS MORE & Jalpaiguri & N26 $52.846^{\prime \prime}$, E $088^{\circ} 56.868^{\prime \prime}$ & 663 & June \\
\hline 28 & NEW GLENCO & Jalpaiguri & N26 $51.963^{\prime \prime}$, E $088^{\circ} 42.802^{\prime \prime}$ & 478 & August, November \\
\hline 29 & NYA SHELLEY & Jalpaiguri & N26 56.626", E $088^{\circ} 54.693^{\prime \prime}$ & 1179 & November \\
\hline 30 & BHARNOBARI & Alipurduar & $\mathrm{N} 26^{\circ} 44.735$, E $089^{\circ} 21.811^{\prime \prime}$ & 472 & June \\
\hline 31 & SATALI & Alipurduar & N $26^{\circ} 43.904$, E $089^{\circ} 21.773^{\prime \prime}$ & 442 & June \\
\hline 32 & BEECH TG & Alipurduar & $\mathrm{N} 26^{\circ} 45.807, \mathrm{E} 089^{\circ} 21.563^{\prime \prime}$ & 502 & June \\
\hline 33 & MAHUA & Alipurduar & $\begin{array}{l}\mathrm{N} 26^{\circ} 48.947^{\prime \prime}, \text { E } 089^{\circ} 22.457^{\prime \prime} \mathrm{N} 26^{\circ} \\
49.044 \text {, E } 089^{\circ} 21.850^{\prime \prime}\end{array}$ & $\begin{array}{l}629 \\
589\end{array}$ & $\begin{array}{l}\text { Jan-Feb, December, } \\
\text { November,July,June, }\end{array}$ \\
\hline 34 & MADHU & Alipurduar & $\mathrm{N} 26^{\circ} 42.709^{\prime \prime}, \mathrm{E} 089^{\circ} 23.064^{\prime \prime}$ & 398 & November \\
\hline 35 & SUBHASINI & Alipurduar & N $26^{\circ} 41.316^{\prime \prime}$, E $089^{\circ} 20.241^{\prime \prime}$ & 306 & November \\
\hline 36 & KALCHINI & Alipurduar & N $26^{\circ} 41.565^{\prime \prime}$, E $089^{\circ} 25.320^{\prime \prime}$ & 420 & December, January \\
\hline 37 & CHUAPARA & Alipurduar & $\mathrm{N} 26^{\circ} 44.380^{\prime \prime}, \mathrm{E} 089^{\circ} 26.394^{\prime \prime}$ & 517 & December \\
\hline 38 & MECHPARA & Alipurduar & $\mathrm{N} 26^{\circ} 43.125^{\prime \prime}, \mathrm{E} 089^{\circ} 26.610^{\prime \prime}$ & 495 & December \\
\hline 39 & RADHARANI & Alipurduar & $\mathrm{N} 26^{\circ} 45.936^{\prime \prime}, \mathrm{E} 089^{\circ} 26.646^{\prime \prime}$ & 625 & December \\
\hline 40 & ATIABARI & Alipurduar & N $26^{\circ} 38.904^{\prime \prime}$, E $089^{\circ} 27.093^{\prime \prime}$ & 287 & December \\
\hline 41 & TOORSA & Alipurduar & $\mathrm{N} 26^{\circ} 49.557^{\prime \prime}, \mathrm{E} 089^{\circ} 22.956^{\prime \prime}$ & 657 & December \\
\hline 42 & BHATKAWA & Alipurduar & $\mathrm{N} 26^{\circ} 40.243^{\prime \prime}$, E $089^{\circ} 26.074^{\prime \prime}$ & 348 & December \\
\hline 43 & DIMA & Alipurduar & $\mathrm{N} 26^{\circ} 40.230^{\prime \prime}, \mathrm{E} 089^{\circ} 26.043^{\prime \prime}$ & 345 & December, January \\
\hline 44 & PATKAPARA & Alipurduar & N 26ㅇ 33.489", E $089^{\circ} 26.069^{\prime \prime}$ & 239 & December \\
\hline 45 & NIMTI JHORA & Alipurduar & N $26^{\circ} 34.927^{\prime \prime}$, E $089^{\circ} 25.744^{\prime \prime}$ & 229 & December,November \\
\hline 46 & MAJHER DABRI & Alipurduar & $\begin{array}{l}\mathrm{N} 26^{\circ} 32.839^{\prime \prime}, \text { E } 089^{\circ} 33.003^{\prime \prime} \mathrm{N} 26^{\circ} \\
33.330^{\prime \prime}, \text { E } 089^{\circ} 30.638^{\prime \prime}\end{array}$ & $\begin{array}{l}151 \\
127\end{array}$ & November \\
\hline 47 & KOHINOOR & Alipurduar & N26 $33.498^{\prime \prime}$, E $089^{\circ} 41.087^{\prime \prime}$ & 162 & November \\
\hline 48 & KARTIKA & Alipurduar & N26 38.747", E $089^{\circ} 43.438^{\prime \prime}$ & 299 & November \\
\hline 49 & RAHIMA BAD & Alipurduar & N26 $40.278^{\prime \prime}$, E $089^{\circ} 43.036^{\prime \prime}$ & 351 & November \\
\hline 50 & TURTURI & Alipurduar & N26 $40.274^{\prime \prime}$, E $089^{\circ} 43.517^{\prime \prime}$ & 379 & November \\
\hline 51 & SRINATH PUR & Alipurduar & N26 $32.626^{\prime \prime}$ E $089^{\circ} 37.168^{\prime \prime}$ & 165 & November \\
\hline 52 & DHOWLA JHORA & Alipurduar & N26 $34.283^{\prime \prime}$, E $089^{\circ} 42.112^{\prime \prime}$ & 206 & November \\
\hline
\end{tabular}




\begin{tabular}{|c|c|c|c|c|c|}
\hline 53 & SANKOSH TEA ESTATE & Alipurduar & N26039.463" E $089^{0} 52.216^{\prime \prime}$ & 306 & December \\
\hline 54 & BHATPARA & Alipurduar & $\mathrm{N} 26^{0} 45.455^{\prime \prime}$ E $089^{0} 28.311^{\prime \prime}$ & 609 & December \\
\hline 55 & NEW LANDS & Alipurduar & 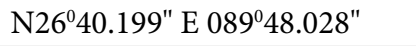 & 316 & December \\
\hline 56 & COOCHBEHAR TEA & Cooch Behar & N260029.526" E $089^{0} 12.273^{\prime \prime}$ & 174 & December \\
\hline 57 & KUMAI TEA ESTATE & Darjeeling & N26 $58.901^{\prime \prime}$ E $088^{0} 50.468^{\prime \prime}$ & 1090 & December \\
\hline 58 & THURBO & Darjeeling & N $26^{\circ} 53.890^{\prime \prime}$, E $088^{\circ} 10.106^{\prime \prime}$ & 5111 & June \\
\hline 59 & OKAYTI & Darjeeling & N $26^{\circ} 54.461^{\prime \prime}$, E $088^{\circ} 08.916^{\prime \prime}$ & 5816 & June \\
\hline 60 & SEEYOK & Darjeeling & N26 55.995", E088 09.321" & 4947 & June \\
\hline 61 & GYABAREE & Darjeeling & $\mathrm{N} 26^{\circ} 49.975^{\prime \prime}$, E $088^{\circ} 13.745^{\prime \prime}$ & 2528 & June \\
\hline 62 & LONGVIEW & Darjeeling & N26 $48.404^{\prime \prime}$, E088 $15.282^{\prime \prime}$ & 1003 & June \\
\hline 63 & MARIONBARI & Darjeeling & N $26^{\circ} 48.562^{\prime \prime}$ E $088^{\circ} 16.128^{\prime \prime}$ & 933 & June \\
\hline 64 & SINGBULLI & Darjeeling & N 26051.009", E $088^{0} 13.336^{\prime \prime}$ & 1100 & June \\
\hline 65 & HAPPY VALLEY & Darjeeling & N $27^{\circ} 03.070^{\prime \prime}$ E $088^{\circ} 15.586^{\prime \prime}$ & 6630 & June \\
\hline 66 & TINGLING & Darjeeling & N26 $50.726^{\prime \prime}$, E $088^{\circ} 11.623^{\prime \prime}$ & 4178 & June \\
\hline 67 & SOURENI & Darjeeling & $\begin{array}{l}\text { N26 } 50.726^{\prime \prime}, \text { E } 088^{\circ} 11.623^{\prime \prime} \\
\text { N } 26^{\circ} 51.413^{\prime \prime} \text { E } 088^{\circ} 11.671^{\prime \prime}\end{array}$ & $\begin{array}{l}4178 \\
4434\end{array}$ & June \\
\hline 68 & PHUGURI & Darjeeling & N26 50.726", E $088^{\circ} 11.623^{\prime \prime}$ & 4178 & June \\
\hline 69 & AMBIOKE & Darjeeling & N26 59.164", E 088 41.840" & 1551 & June \\
\hline 70 & YOUNTANG & Darjeeling & N $26^{\circ} 58.246^{\prime \prime}$ E $088^{\circ} 49.651^{\prime \prime}$ & 1203 & November \\
\hline 71 & ZURRANTEE & Darjeeling & N $26^{\circ} 56.997^{\prime \prime}$ E $088^{\circ} 45.658^{\prime \prime}$ & 1037 & November \\
\hline 72 & ENGO & Darjeeling & N $26^{\circ} 57.283^{\prime \prime}$, E $088^{\circ} 46.047^{\prime \prime}$ & 1130 & November \\
\hline 73 & NAGAISUREE & Darjeeling & N $26^{\circ} 56.152^{\prime \prime}$, E $088^{\circ} 47.013^{\prime \prime}$ & 830 & November \\
\hline 74 & INDONG & Darjeeling & N $26^{\circ} 54.376^{\prime \prime}$ E $088^{\circ} 46.509^{\prime \prime}$ & 815 & November \\
\hline
\end{tabular}

\section{Order LEPIDOPTERA}

Butterflies, moths and skippers are one of the most important groups of insect in the animal world under the order Lepidoptera. They are beautiful in colours, diverse in their numbers, and play a pivotal role in the faunal analysis and environmental monitoring and planning of any region. The role of butterflies and some moths in any habitat is best known by even common people nowadays, as good and potential natural pollinators, ecological indicators etc., which enable to assess or monitor habitat changes. Among insects, butterflies are well studied groups in terms of their taxonomy, geographic distribution etc., and therefore, they are the suitable group for ecological studies, Butterflies show distinct pattern of habitat utilization. The presence of butterflies denotes the availability of larval food plants in great abundance. Butterfly host plants are those plants, on which specific butterfly species lay eggs, and caterpillars will then hatch and use plant as their sole food source. Butterflies are generally diurnal and visit flowers that open in the morning and also during the hours of bright sunshine.

Presently 37 species belonging to 06 families of butterflies from North Bengal tea gardens were collected and identified. Of them, Nymphalidae shares 20 species followed by Pieridae ( 10 species), Hesperiidae ( 03 species), Papilionidae (02 species), Lycaenidae (01 species) and Riodinidae (01 species) (Table 2).

Table 2. List of Butterflies associated with tea plants of North Bengal

\begin{tabular}{|l|l|}
\hline No. & Species \\
\hline & Family HESPERIIDAE \\
\hline 1 & Celaenorrhinus leucocera (Kollar, 1844) \\
\hline 2 & Pelopidas mathias (Fabricius, 1798) \\
\hline 3 & Tagiades japeteus attieus (Fabricius, 1793) \\
\hline & Family LYCAENIDAE \\
\hline 4 & Poritia hewitsoni Moore, 1865 \\
\hline
\end{tabular}




\begin{tabular}{|l|l|}
\hline & Family NYMPHALIDAE \\
\hline 5 & Aglais cshmirensis (Kollar, 1848) \\
\hline 6 & Athyma kanwa Moore, 1858 \\
\hline 7 & Athyma perius (Linnaeus, 1758) \\
\hline 8 & Danaus chrysippus (Linnaeus, 1758) \\
\hline 9 & Danaus genutia (Cramer, 1779) \\
\hline 10 & Euploea core core (Cramer, 1780) \\
\hline 11 & Euploea mulciber mulciber (Cramer, 1777) \\
\hline 12 & Junonia almana Linnaeus, 1758 \\
\hline 13 & Junonia atlites Linnaeus, 1763 \\
\hline 14 & Junonia hierta (Fabricius, 1798) \\
\hline 15 & Junonia iphita Cramer, 1779 \\
\hline 16 & Junonia lemonias (Linnaeus, 1758) \\
\hline 17 & Melanitis leda (Linnaeus, 1758) \\
\hline 18 & Mycalesis perseus blasius (Fabricius, 1798) \\
\hline 19 & Neptis hylas astola Linnaeus, 1872 \\
\hline 20 & Parantica aglea melanoides Moore, 1883 \\
\hline 21 & Symbrenthia hippoclus (Cramer, 1782) \\
\hline 22 & Tirumala hamata septentrionis (Butler, 1874) \\
\hline 23 & Ypthima hubneri Kirby, 1871 \\
\hline 24 & Ypthima similis Elwis \& Edward, 1893 \\
\hline & Family PAPILIONIDAE \\
\hline 25 & Papilio polytes stichius Evans, 1912 \\
\hline 26 & Parides dasarada (Moore, 1857) \\
\hline & Family PIERIDAE \\
\hline 27 & Appias libythea (Fabricius, 1775) \\
\hline 28 & Catopsilia crocale Cramer, 1775 \\
\hline 29 & Catopsilia pomona (Fabricius, 1775) \\
\hline 30 & Catopsilia pyranthe (Linnaeus, 1758) \\
\hline 31 & Cepora nadina (Lucas, 1852) \\
\hline 32 & Eurema blanda silhetana Wallace, 1867 \\
\hline 33 & Eurema hecabe (Linnaeus, 1758) \\
\hline 34 & Hebomoia glaucippe (Linnaeus, 1758) \\
\hline 35 & Leptosia nina nina (Fabricius, 1739) \\
\hline 36 & Pieris brassicae (Linnaeus, 1758) \\
\hline & Family RIODINIDAE \\
\hline 37 & zemeros flegyas (Guerin, 1843) \\
\hline
\end{tabular}

Beside above, 40 species belonging to 06 families of moths are found associated with tea plants in North Bengal. The family Erebidae dominates with 15 species followed by the family Crambidae (09 species), Geometridae (08 species), Sphingidae (04 species), Zygaenidae (03 species) and
Torticidae (01 species). Present communication reports 20 moth species $\left({ }^{*}\right)$ as tea pests from North Bengal (Table 3 ).

Table 3. List of moths associated with tea plants of North Bengal

\begin{tabular}{|c|c|}
\hline No. & Species \\
\hline & Family CRAMBIDAE \\
\hline 1 & Omiodes surrectalis (Walker, 1866) \\
\hline 2 & Arthroschista hilaralis (Walker, 1859) \\
\hline 3 & Glyphodes stolalis Guenee, 1854 \\
\hline 4 & Parotis marginata (Hampson, 1893) \\
\hline 5 & Herpetogramma licarsisalis (Walker, 1859) \\
\hline 6 & Cnaphalocrocis poeyalis (Boisduval, 1833) \\
\hline 7 & Nosophora semitritalis (Lederer, 1863) \\
\hline 8 & Eoophyla sejuncta (Snellen, 1876 ) \\
\hline \multirow[t]{2}{*}{9} & Diaphania sp. \\
\hline & Family EREBIDAE \\
\hline $10^{*}$ & Arctornis submarginata Walker, 1855 \\
\hline $11^{\star}$ & Somena scintillans (Walker, 1856) \\
\hline $12^{\star}$ & Lymantria marginalis (Walker, 1862) \\
\hline $13^{*}$ & Miltochrista cuneonotata (Walker, 1855) \\
\hline 14 & Chionaema bianca (Walker, 1856) \\
\hline $15^{\star}$ & Nyctemera adversata Schaller, 1788 \\
\hline $16^{*}$ & Creatonotos transiens (Walker, 1855) \\
\hline 17 & Creatonotos gangis (Linnaeus, 1763) \\
\hline $18^{*}$ & Argina argus (Kollar, 1844) \\
\hline 19 & Argina astrea (Drury, 1773) \\
\hline 20 & Asota egens (Walker, 1854) \\
\hline $21^{\star}$ & Arna bipunctapex Hampson, 1891 \\
\hline $22^{*}$ & Euproctis sp. \\
\hline $23^{*}$ & Orgyia sp. \\
\hline \multirow[t]{2}{*}{24} & Callimorpha plagiata Walker, 1855 \\
\hline & Family GEOMETRIDAE \\
\hline $25^{\star}$ & Ascotis selenaria (Denis \& Schiffermiller, 1775) \\
\hline $26^{*}$ & Biston suppressaria (Guenee, 1858) \\
\hline $27^{\star}$ & Hyposidra talaca Walker, 1860 \\
\hline $28^{\star}$ & Hyposidra infixaria Walker, 1860 \\
\hline 29 & Semiothisa eleonora (Villers, 1789) \\
\hline $30^{*}$ & Ectropis sp. \\
\hline $31^{*}$ & Cleora sp. \\
\hline \multirow[t]{2}{*}{$32^{*}$} & Petelia sp. \\
\hline & Family SPHINGIDAE \\
\hline 33 & Theretra nessus Drury, 1773 \\
\hline 34 & Hippotion boerhaviae (Fabricius, 1775) \\
\hline
\end{tabular}




\begin{tabular}{|l|l|}
\hline 35 & Argius convolvuli (Linnaeus, 1758) \\
\hline 36 & Acosmeryx omissa Rothschild and Jordan, 1903 \\
\hline & Family TORTRICIDAE \\
\hline 37 & Loboschiza koenigiana (Fabricius, 1775) \\
\hline & Family ZYGAENIDAE \\
\hline $38^{*}$ & Eterusia aedea aedea Linnaeus, 1763 \\
\hline $39^{*}$ & Eterusia edcola Doubleday, 1847 \\
\hline $40^{*}$ & Trypanophora semihyalina Kollar, 1844 \\
\hline
\end{tabular}

\section{Order HEMIPTERA}

Hemiptera (True Bugs), is a very large and diverse order and also important in agriculture, known to cause direct damage to plants by herbivory and indirectly by transporting diseases. Predatory Hemiptera have also been used in agricultural systems to control pests

29 species belonging to 28 genera of hemipteran bugs from the tea gardens of North Bengal were reported. Of them, the family Pentatomidae shares the highest number of species (07), followed by Coreidae (04), Lygaeidae (03), Fulgoridae (03), Reduviidae (03), Cicadidae (02), Cercopidae (01), Membracidae (01), Largidae (01), Pyrrhocoridae (01), Scutelleridae (01), Miridae (01). 05 hemipteran species $\left({ }^{*}\right)$ were reported as pest of tea from North Bengal (Table 4).

Table 4. List of hemipteran species associated with tea plants of North Bengal

\begin{tabular}{|l|l|}
\hline No. & Species \\
\hline & Family CERCOPIDAE \\
\hline 1 & Leptataspis fulviceps (Dallas, 1850) \\
\hline & Family CICADIDAE \\
\hline $2^{*}$ & Huechys sanguinea (De Geer, 1773) \\
\hline 3 & Paomponia picta (Walker, 1870) \\
\hline 4 & Lamily FULGORIDAE \\
\hline 5 & Ricania speculum (Walker, 1851) \\
\hline 6 & Polydictya tricolor (Westwood, 1845) \\
\hline & Family MEMBRACIDAE \\
\hline 7 & Darthula hardwicki (Gray, 1831) \\
\hline & Family COREIDAE \\
\hline 8 & Cletus bipunctatus (Herrich-Schaffer, 1840) \\
\hline 9 & Riptortus linearis (Fabricius, 1775) \\
\hline 10 & Riptortus pedestris (Fabricius, 1775) \\
\hline $11^{*}$ & Elasmomia granulipes (Westwood, 1842) \\
\hline
\end{tabular}

\begin{tabular}{|l|l|}
\hline & Family LYGAEIDAE \\
\hline 12 & Paromius exiguous (Distant, 1883) \\
\hline 13 & Metochus uniguttatus (Thunberg, 1879) \\
\hline 14 & Graptostethus trisignatus (Distant, 1879) \\
\hline & Family PENTATOMIDAE \\
\hline $15^{*}$ & Halys dentatus (Fabricius, 1775) \\
\hline 16 & Nezara viridula (Linnaeus, 1758) \\
\hline $17^{\star}$ & Andrallus spinidens (Fabricius, 1787) \\
\hline 18 & Tolumnia latipes (Dallas, 1851) \\
\hline 19 & Erthesina fullo (Thunberg, 1783) \\
\hline 20 & Plautia crossata (Stal, 1869) \\
\hline 21 & Eocanthecona furcellata (Wolf, 1811) \\
\hline & Family LARGIDAE \\
\hline 22 & Macroceraria grandis (Gray, 1832) \\
\hline & Family PYRRHOCORIDAE \\
\hline 23 & Odontopus nigricornis (Stall, 1861) \\
\hline 24 & Physopelta quadrigutta (Bergroth, 1894) \\
\hline & Family REDUVIIDAE \\
\hline 25 & Vilius melanopterus (Stall, 1863) \\
\hline 26 & Coranus spiniscutis (Reuter, 1881) \\
\hline 27 & Triatoma rubrofasciatus (De Geer, 1773) \\
\hline & Family SCUTELLERIDAE \\
\hline 28 & Cantao ocellatus (Thunberg, 1784) \\
\hline & Family MIRIDAE \\
\hline $29^{*}$ & Helopeltis theivora (Waterhouse, 1886) \\
\hline
\end{tabular}

\section{Order DIPTERA}

The Diptera are commonly known as "True flies" and include many familiar insects such as mosquitoes, flies (black flies, fruit flies, blow flies, house flies etc.), midges (biting, non-biting), gnats (fungus, root), keds, bots etc. This diversity of names denotes the importance of the group and reflects the range of organisms in the order. Diptera exploit most of the available ecological niches in most of the biological roles, e.g., saprophages (many), commensals (some Scatopsidae, Chironomidae and Phoridae), predators (many), parasites (many), symbionts (some Chironomidae and algae) and scavengers.

24 species under 23 genera of (08) families of Diptera were reported from the tea gardens of North Bengal (Table 5). Of them, the family Syrphidae shares maximum number of species (09), followed by Asilidae (04), Bombyllidae (02), Calliphoridae (02), Rhiniidae (02), 
Muscidae (02) and Sarcophagidae (02) and Tipulidae (01). Shah and Mitra (2015) reports Microstyllum pseudoanantakrishnanii Joseph and Parui as the predator of the moths from the tea gardens of North Bengal $\left.{ }^{* *}\right)$. Das et al. (2010) confirmed 54 species of predators of insect pests from tea gardens of northern West Bengal, of which a single dipteran species Ischiodon scutellaris ${ }^{* *}$ ) as a predator (maggot stage) of tea pest (Table 5).

Table 5. List of dipteran species associated with tea plants of North Bengal

\begin{tabular}{|c|c|}
\hline No. & Species \\
\hline & Family TIPULIDAE \\
\hline \multirow[t]{2}{*}{1} & Nephrotoma consimilis (Brunetti, 1911) \\
\hline & Family ASILIDAE \\
\hline $2^{* *}$ & $\begin{array}{l}\text { Microstyllum pseudoanantakrishnanii (Joseph \& Parui, } \\
\text { 1982) }\end{array}$ \\
\hline 3 & Microstyllum brunnipenne (Macquart, 1849) \\
\hline 4 & Cophinopoda chinensis (Fabricius, 1794) \\
\hline \multirow[t]{2}{*}{5} & Promachus duvaucelii (Macquart, 1838) \\
\hline & Family SYRPHIDAE \\
\hline 6 & Volucella sp. \\
\hline 7 & Episyrphus balteatus (De Geer, 1776) \\
\hline 8 & Eristalis tenax (Linnaeus, 1758) \\
\hline 9 & Mesembrias sp. \\
\hline 10 & Eristalodes paria (Bigot, 1880) \\
\hline 11 & Eristalinus arvorum (Fabricius, 1787) \\
\hline 12 & Melanostoma orientale (Wiedemann, 1824) \\
\hline 13 & Betasyrphus serarius (Wiedemann, 1830) \\
\hline \multirow[t]{2}{*}{$14^{* *}$} & Ischiodon scutellaris (Fabricius, 1805) \\
\hline & Family BOMBYLIIDAE \\
\hline 15 & Hyperalonia suffusipennis (Brunetti, 1909) \\
\hline \multirow[t]{2}{*}{16} & Exoprosopa (Exoprosopa) insulata (Walker, 1852) \\
\hline & Family CALLIPHORIDAE \\
\hline 17 & Chrysomya megacephala (Fabricius, 1794) \\
\hline \multirow[t]{2}{*}{18} & Hemipyrellia ligurriens (Wiedemann, 1830) \\
\hline & Family RHINIIDAE \\
\hline 19 & Idiella mandarina (Wiedemann, 1830) \\
\hline \multirow[t]{2}{*}{20} & Stomorhina discolor (Fabricius, 1794) \\
\hline & Family MUSCIDAE \\
\hline 21 & Musca (Musca) domestica (Linnaeus, 1758) \\
\hline \multirow[t]{2}{*}{22} & Neomyia indica (Robineau-Desvoidy, 1830) \\
\hline & Family SARCOPHAGIDAE \\
\hline 23 & Sarcophaga (Liosarcophaga) dux (Thomson, 1869) \\
\hline 24 & Sarcophaga sp. \\
\hline
\end{tabular}

\section{Order COLEOPTERA}

Coleoptera (beetles and weevils) is the largest order in the class Insecta. They feed on a wide variety of diets, inhabit all terrestrial and fresh-water environments, and exhibit a number of different life styles. Many species are herbivores, adapted to feed on the roots, stems, leaves, or reproductive structures of their host plants. Some species live on fungi, others burrow into plant tissues and some excavate tunnels in wood or under bark. Many beetles are predators. Some beetles are scavengers, feeding primarily on carrion, decaying wood or other dead organic matter.

19 species under 16 genera belonging to 05 families of the order Coleoptera were reported from the tea gardens of North Bengal (Table 6). Of them, Scarabaeidae represent 09 Species followed by Cerambycidae (06 Species), Coccinellidae (02 Species), Lucanidae (01 species) and Curculionidae (01 species). Among the family Scarabaeidae, three species namely, Holotrichia sp., Anomala dimidiata and Xylotrupes gideon are reported as pests of tea (Roy et al., 2014).

Only two species of the family Cocinellidae, Micraspis discolor and Coccinella septempunctata are recorded during present study (Table 6). Micraspis discolor is the most abundant species of coccinellid in all the ecosystems. Roy et al., (2010) were observed twenty species of coccinellid predators in Sub-Himalayan tea plantation of North Bengal during 2004 to 2006. Of these, Micraspis discolor ${ }^{* *}$ ) was dominant $(42.5 \%)$ in the conventionally managed tea plantations. The life cycle studies also suggested that the tea aphid was the preferred prey for Micraspis discolor, but the predator can survive on red spider mite also Roy et al., (2010). The abundance of adult $M$. discolor in rice at flowering phase does not correspond to prey abundance in the field but rather reflects an inclination to pollen feeding more than entomophagy (Shanker et al. 2013). C. septempunctata ${ }^{* *}$ ) has a broad ecological range. Both the adults and the larvae are voracious predators of aphids. The diversity of lady bird beetles in tea ecosystem may be due to the complex and stable ecosystem of tea plantations.

05 species $\left(^{*}\right)$ as pest and 02 species as predators $\left({ }^{* *}\right)$ of the order Coleoptera were reported from the tea gardens of North Bengal (Table 6). 
Table 6. List of coleopteran species associated with tea plants of North Bengal

\begin{tabular}{|l|l|}
\hline No. & Species \\
\hline & Family CERAMBYCIDAE \\
\hline 1 & Dorysthenes (Lophosternus) indicus (Hope, 1831) \\
\hline 2 & Dorysthenes (Paraphrus) granulosus (Thomson, 1861) \\
\hline 3 & Xystrocera globosa (Olivier, 1795) \\
\hline 4 & Aristobia approximator (Thomson, 1865) \\
\hline 5 & Batocera rufomaculata (De Geer, 1775) \\
\hline 6 & Nupserha sp. \\
\hline & Family SCARABAEIDAE \\
\hline $7^{\star}$ & Xylotrupes gideon (Fabricius, 1775) \\
\hline $8^{\star}$ & Catharsius molossus (Linnaeus, 1758) \\
\hline 9 & Catharsius sagax (Quenstedt, 1806) \\
\hline 10 & Onitis subopacus (Arrow, 1931) \\
\hline $11^{*}$ & Holotrichia sp. \\
\hline 12 & Brahmina sp. \\
\hline 13 & Anomala grandis (Hope, 1840) \\
\hline $14^{*}$ & Anomala dimidiata (Hope, 1831) \\
\hline 15 & Melolontha guttigera (Sharp, 1876) \\
\hline & Family COCCINELLIDAE \\
\hline $16^{* *}$ & Micraspis discolor (Fabricius, 1798) \\
\hline $17^{* *}$ & Coccinella septempunctata (Linnaeus,1758) \\
\hline & Family LUCANIDAE \\
\hline 18 & Odontolabis siva (Hope \& Westwood, 1845) \\
\hline $19^{*}$ & Family CURCULIONIDAE \\
\hline
\end{tabular}

\section{Order ODONATA}

The Odonata is an important insect order which comprises of fascinating and harmless insects popularly known as dragonflies (Anisoptera) and damsel flies (Zygoptera). Odonates are amphibiotic and diverse in nature. Many of these species inhabit wide variety of natural fresh water habitats, though there are some species which have adapted themselves to man-made water bodies. Being habitat specific and sensitive to habitat change their presence or absence indicates the quality of an ecosystem. They are considered as beneficial insects because their larvae (aquatic) and adults (terrestrial) are predators. Thus, assist in control of insect pest populations.

12 species under 08 genera belonging to 02 families of the order Odonata were reported from different tea gardens of North Bengal. The family Libellulidae shared
09 species and the family Coenagrionidae shared 03 species.

Das et al. (2010) observed that in sub Himalayan tea plantations of North Bengal, $4 \%$ of the predatory insects comprised of odonates. Present study documented Orthetrum sabina (Drury) ${ }^{* *}$ ) predates on the Hyposidra talaca (Walker) in the tea gardens of North Bengal (Table 7).

Table 7. List of odonate species associated with tea plants of North Bengal

\begin{tabular}{|l|l|}
\hline & Family LIBELLULIDAE \\
\hline 1 & Crocothemis erythraea (Brulle, 1832) \\
\hline 2 & Crocothemis servilia (Drury, 1773) \\
\hline 3 & Diplacodes trivialis (Rambur, 1842) \\
\hline 4 & Neurothemis fulvia (Drury, 1773) \\
\hline 5 & Orthetrum glaucum (Brauer, 1865) \\
\hline 6 & Orthetrum pruinosum (Burrneister, 1839) \\
\hline $7^{* *}$ & Orthetrum sabina (Drury, 1770) \\
\hline 8 & Pantala flavescens (Fabricius, 1798) \\
\hline 9 & Trithemis festiva (Rambur, 1842) \\
\hline & Family COENAGRIONIDAE \\
\hline 10 & Ischnura aurora (Brauer, 1865) \\
\hline 11 & Pseudagrion microcephalum (Rambur, 1842) \\
\hline 12 & Pseudagrion rubriceps (Selys, 1876) \\
\hline
\end{tabular}

\section{Order ORTHOPTERA}

The insects under order Orthoptera are popularly known as grasshoppers, crickets etc. These insects inhabit many variety of habitats, like open grasslands, gardens, pastures, agro-fields, swamps and rain forests etc. Present communication reports 06 species under 06 genera belonging to 03 families of Orthoptera from the tea gardens of North Bengal (Table 8 ). Of them, Tettigonidae shares highest number of species (04) followed by Gryllidae and Pyrgomorphidae one species each. Nath et al., (2009) has been detected Atractomorpha crenulata (Fabricius, 1793) as possible pest of tea plant and other crops. Present communication reports only four orthopteran species $\left(^{*}\right)$ as pest of tea plant from North Bengal (Table 8).

Table 8. List of orthopteran species associated with tea plants of North Bengal

\begin{tabular}{|l|l|}
\hline No. & Species \\
\hline & Family TETTIGONIDAE \\
\hline $1^{\star}$ & Holochlora indica (Kirby, 1906) \\
\hline
\end{tabular}




\begin{tabular}{|l|l|}
\hline 2 & Ducetia japonica (Thunberg, 1815) \\
\hline 3 & Euconocephalus pallidus (Redtenbacher, 1891) \\
\hline $4^{*}$ & Mecopoda elongata (Linnaeus, 1758) \\
\hline & Family GRYLLIDAE \\
\hline $5^{*}$ & Tarbinskiellus orientalis (Fabricius, 1775) \\
\hline & Family PYRGOMORPHIDAE \\
\hline $6^{*}$ & Atractomorpha crenulata (Fabricius, 1793) \\
\hline
\end{tabular}

\section{Discussion}

Tea is grown as a perennial monoculture crop over large contiguous areas in different parts of India. These tea gardens itself occupy a wide variety of functional niches and microhabitats. Insects, the most diverse component of tea gardens play a major role in the sustainable and healthy functioning of tea ecosystem. The tea estates of
Assam and North Bengal are well known for the pest infestation since time immemorial. The tea garden owners/ researcher/ managers are only interested to high yields and control of the major pests. Therefore, there conventional tea cultivation has often accomplished high yields and stable crop production which need more use of chemical pesticides. These ultimately lead resistance, resurgence of pests and destruction of natural enemies.

In addition to this, there is very limited taxonomic knowledge on other group of insects who are also part and parcel of this tea ecosystem. In fact, there number is more and playing vital role in maintaining ecosystem healthy and sustainable. Keeping this in view, the present study was conducted in 74 tea gardens to identify the tea plant associated insects and their functional role in tea garden ecosystem.

Table 9. List of pest, predator and pollinators species of other plants reported from the tea ecosystem of North Bengal

\begin{tabular}{|c|c|c|}
\hline \multicolumn{3}{|c|}{ Order LEPIDOPTERA } \\
\hline No & Species & Biological Role \\
\hline \multicolumn{3}{|c|}{ Family SPHINGIDAE } \\
\hline 1 & Theretra nessus (Drury, 1773) & Dioscorea (family Dioscoreaceae), Amarantahceae, Cucurbitaceae \\
\hline 2 & Hippotion boerhaviae (Fabricius, 1775) & Amarantahceae, Cucurbitaceae, Rubiaceae \\
\hline 3 & Argius convolvuli (Linnaeus, 1758) & $\begin{array}{l}\text { Arachis hypogaea (groundnut), Chrysanthemum (daisy), Colocasia esculenta } \\
\text { (taro), Helianthus annuus (sunflower), Ipomoea batatas (sweet potato), } \\
\text { Vigna mungo (black gram), Vignara diata (mung bean), Vigna unguiculata } \\
\text { (cowpea), Vigna vexillata (wild sweet pea) }\end{array}$ \\
\hline \multicolumn{3}{|c|}{ Family GEOMETRIDAE } \\
\hline 4 & Semiothisa eleonora (Villers, 1789) & Pest of Mimosa rubicaulis and Acacia concinna \\
\hline \multicolumn{3}{|c|}{ Family PIERIDAE } \\
\hline 5 & Catopsilia pyranthe (Linnaeus, 1758) & Pests of crops \\
\hline 6 & Eurema blanda silhetana (Wallace, 1867) & Pest of shade trees of tea plantations \\
\hline 7 & Eurema hecabe (Linnaeus, 1758) & Pests of timber plants \\
\hline 8 & Pieris brassicae (Linnaeus, 1758) & Pest of Cruciferous plants \\
\hline \multicolumn{3}{|c|}{ Family LYCAENIDAE } \\
\hline 9 & Poritia hewitsoni (Moore, 1865) & Pest of Sal forests \\
\hline \multicolumn{3}{|c|}{ Order HEMIPTERA } \\
\hline \multicolumn{3}{|c|}{ Family CERCOPIDAE } \\
\hline 10 & Leptataspis fulviceps (Dallas, 1850) & Phytopgagus \\
\hline \multicolumn{3}{|c|}{ Family CICADIDAE } \\
\hline 11 & Paomponia fusca (Olivier, 1790) & Phytophagus \\
\hline \multicolumn{3}{|c|}{ Family FULGORIDAE } \\
\hline 12 & Lawana conspersa (Walker, 1851) & Phytophagus \\
\hline 13 & Ricania speculum (Walker, 1851) & Oil Palm \\
\hline
\end{tabular}




\begin{tabular}{|c|c|c|}
\hline \multicolumn{3}{|c|}{ Family COREIDAE } \\
\hline 14 & Cletus bipunctatus (Herrich-Schaffer, 1840) & Amaranthus viridis L. Portula caoleracea L. \\
\hline & Riptortus linearis (Fabricius, 1775) & $\begin{array}{l}\text { Maize, Millets, Pulses, Sorghum, } \\
\text { Soyabean, Sweet Potato and Murraya koenigii. }\end{array}$ \\
\hline 15 & Riptortus pedestris (Fabricius, 1775) & Mung Bean, legume crops \\
\hline \multicolumn{3}{|c|}{ Family LYGAEIDAE } \\
\hline 16 & Paromius exiguous (Distant, 1883) & Rice \\
\hline 17 & Graptostethus trisignatus (Distant, 1879) & Phytophagus \\
\hline \multicolumn{3}{|c|}{ Family PENTATOMIDAE } \\
\hline 18 & Tolumnia latipes (Dallas, 1851) & $\begin{array}{l}\text { Vigna unguiculatas esquipedalis, Sanatalum album, Solanum nigrum and } \\
\text { Lantana sp. }\end{array}$ \\
\hline 19 & Eocanthecona furcellata (Wolff, 1811) & Predator \\
\hline \multicolumn{3}{|c|}{ Family LARGIDAE } \\
\hline 20 & Macrocheraia grandis (Gray, 1832) & Cotton, okra, hibiscus, Trewia nudiflora, Bombax ceiba, etc. \\
\hline \multicolumn{3}{|c|}{ Family PYRRHOCORIDAE } \\
\hline 21 & Odontopus nigricornis (Stal, 1861) & Sterculia foetida, Acacia mangium, , Acacia auriculiformis \\
\hline 22 & Physopelta quadrigutta (Bergroth, 1894) & Phytophagous \\
\hline \multicolumn{3}{|c|}{ Family REDUVIIDAE } \\
\hline 23 & Vilius melanopterus (Stal, 1863) & Mainly ants, bes, termites \\
\hline 24 & Coranus spiniscutis (Reuter, 1881) & Soft bodied insects, catterpiller, termites \\
\hline 25 & Triatoma rubrofasciatus (De Geer, 1773) & Haematophagous \\
\hline \multicolumn{3}{|c|}{ Order DIPTERA } \\
\hline \multicolumn{3}{|c|}{ Family ASILIDAE } \\
\hline 26 & Microstyllum brunnipenne (Macquart, 1849) & Predator \\
\hline 27 & Cophinopoda chinensis (Fabricius, 1794) & Predator \\
\hline 28 & Promachus duvaucelii (Macquart, 1838) & Predator \\
\hline \multicolumn{3}{|c|}{ Family SYRPHIDAE } \\
\hline 29 & Volucella sp. & Flower visitors of tea plants \\
\hline 30 & Eristalis tenax (Linnaeus, 1758) & Effective pollinators of different plant species. \\
\hline 31 & Mesembrias sp. & Flower visitor of different plants. \\
\hline 32 & Eristalodes paria (Bigot, 1880) & Flower visitor of Helianthus sp. \\
\hline 33 & Eristalinus arvorum (Fabricius, 1787) & Flower visitor of several flowers. \\
\hline 34 & Melanostoma orientale (Wiedemann, 1824) & Effective pollinator of Brassica juncea. \\
\hline \multicolumn{3}{|c|}{ Family BOMBYLIIDAE } \\
\hline 35 & Hyperalonia suffusipennis (Brunetti, 1909) & Predator \\
\hline 36 & $\begin{array}{l}\text { Exoprosopa (Exoprosopa) insulate (Walker, } \\
1852 \text { ) }\end{array}$ & Effective pollinator of some medicinal plants in India. \\
\hline \multicolumn{3}{|c|}{ Family CALLIPHORIDAE } \\
\hline 37 & Chrysomya megacephala (Fabricius, 1794) & Flower visitor \\
\hline 38 & Hemipyrellia ligurriens (Wiedemann, 1830) & Pollinator of Periscaria chinensis. \\
\hline
\end{tabular}




\begin{tabular}{|c|c|c|}
\hline \multicolumn{3}{|c|}{ Family RHINIIDAE } \\
\hline 39 & Idiella mandarina (Wiedemann, 1830) & Flower visitor \\
\hline 40 & Stomorhina discolour (Fabricius, 1794) & Flower visitor of Brassica juncea. \\
\hline \multicolumn{3}{|c|}{ Family MUSCIDAE } \\
\hline 41 & Musca (Musca) domestica (Linnaeus, 1758) & Most common flower visitor \\
\hline 42 & Neomyia indica (Robineau-Desvoidy, 1830) & Flower visitors of Alstonia scholaris and Helianthus annuus. \\
\hline \multicolumn{3}{|c|}{ Order COLEOPTERA } \\
\hline \multicolumn{3}{|c|}{ Family CERAMBYCIDAE } \\
\hline 43 & $\begin{array}{l}\text { Dorysthenes (Lophosternus) indicus (Hope, } \\
1831 \text { ) }\end{array}$ & Root borer \\
\hline 44 & $\begin{array}{l}\text { Dorysthenes (Paraphrus) granulosus } \\
\text { (Thomson, 1861) }\end{array}$ & Root borer \\
\hline 45 & Xystrocera globosa (Olivier, 1795) & Pest of shade tree of tea garden \\
\hline 46 & Aristobia approximator (Thomson, 1865) & Borers of dead log \\
\hline 47 & Batocera rufomaculata (De Geer, 1775) & Borers of dead log \\
\hline 48 & Nupserha sp. & Borers of dead log \\
\hline \multicolumn{3}{|c|}{ Family SCARABAEIDAE } \\
\hline 49 & Onitis subopacus Arrow. 1931 & Coprophagous \\
\hline 50 & Holotrichia sp. & Coprophagous \\
\hline 51 & Brahmina sp. & Root of Young Tea and Seedling Plants \\
\hline 52 & Anomala grandis (Hope, 1840) & Phytophagous \\
\hline \multicolumn{3}{|c|}{ Family LUCANIDAE } \\
\hline 53 & Odontolabis siva (Hope \& Westwood, 1845) & Root borer \\
\hline
\end{tabular}

In this research work, 167 species belonging to 139 genera of 42 families under 06 order of insects from the tea gardens of North Bengal. Of them, Lepidoptera contained the highest number of species (77), followed by Hemiptera (29), Diptera (24), Coleoptera (19), Odonata (12) and Orthoptera (06).

Among the order Lepidoptera, 37 species are butterflies reported from these tea gardens. According to Krenn (2010), the majority of butterflies feed on floral nectar, though their role as effective pollinators remains doubtful in many examples (Courtney et al., 1982; Wiklund, 1981; Wiklund et al., 1979). In fact, some authors regard butterflies as opportunistic flower visitors that use resources as they become available during the season (Shreeve, 1992; Stefanescu and Traveset, 2009; Tudor et al., 2004). Besides this, some butterflies reported from Tea ecosystems are considered as pest of other plants. Chaudhary and Saravanan (2013) reported Catopsilia pyranthe (Linnaeus, 1758) as one of the major pests of lndian senna (Cassia angustifolia Yehl). Jha and
Sen Sharma (2008) reported Eurema hecabe (Linnaeus, 1758) as pest of Tectona grandis. The other butterflies recorded as pest of Tectona grandis by Jha and Sen Sharma (2008) is Poritia hewitsoni (Table 9). In their study, David et al., (2004) recorded Eurema blanda silhetana as pest of shade trees of tea plantations. Similarly, Pieris brassicae reported in this communication is well known pest of Cruciferous plants (Sharma, 2011) (Table 9). Among the 40 species of moths reported during this study period, Arctornis submarginata, Somena scintillans, Lymantria marginalis, Miltochrista cuneonotata, Nyctemera adversata, Creatonotos transiens, Argina argus, Arna bipunctapex, Euproctis sp., Orgyia sp., of the family Erebidae and Ascotis selenaria, Biston suppressaria, Hyposidra talaca, Hyposidra infixaria, Ectropis sp., Cleora sp., Petelia sp. of the family Geometridae are the recognised pests of Tea. Besides this, Eterusia aedea aedea, Eterusia edcola, Trypanophra semihyalina of the family Zygaenidae are also the major threats of Tea gardens in North Bengal (Table 9). Present communication reports 29 species of hemipteran bugs 
from the tea gardens of North Bengal. Of them, 14 species of hemipteran bugs are found as pests and 3 species as carnivore/predators of other crops (Table 9). Ariyarathna et al., (2007) stated that, insufficient knowledge on breeding systems and floral biology is a major barrier in strategic planning of practical tea crop breeding programmes as well as in research on tea crop improvement. As observed, tea flowers being bisexual, bowl shaped, actinormorphic, with large number of stamens, are unspecialised and arranged solitarily or loosely clustered. Faegri and Pijl (1979) reported that such characteristics are typical floral syndromes among insect pollinated plant species. In their study, Bezbaruah (1975), Wickramaratne and Vitarana (1985) showed that, general pollinators, mainly dipterans contribute to pollination success in tea. A study on Camelia sinensis from Sri Lanka by Wickramaratne and Vitarana (1985) reveals that dipteran flies were numerous (60-90\% of total insects) and the efficient pollinators of tea whereas bees only visit seasonally and in very few numbers. Mitra $e t$ al., (2017) are also discussed about their pollination ability on tea plants in North Bengal. Present study recorded 24 species of 8 families from the tea gardens of North Bengal (Table 5). Of them, the syrphids, bombyllids, calliphorids and muscids are potetial pollinators of many crop plants (Table 9). Beside their role as pollination, dipterans are also act as biological control agents. Robber flies (Diptera: Asilidae) are the natural predators to several insect pest for different agricultural crops or plants. Various work reveals robber fly as the predators of spider (Dennis et al., 2012) and insects like Hymenoptera (Dennis and Lavigne, 2007), Lepidoptera, (Dennis et al., 2012), Coleoptera, (Shelly and Pearson, 1978), Orthoptera, (Joern and Rudd, 1982) and Hemiptera (Dennis et al., 2010). In this communication, 3 species of robber flies, viz. Microstyllum brunnipenne Macquart, 1849, Cophinopoda chinensis (Fabricius, 1794) and Promachus duvaucelii (Macquart, 1838) are reported which are carnivores and predators (Table 9). Among five families of the order Coleoptera from the tea gardens of North Bengal scarabs are the dominant group found during this study. Adults of this beetle generally feed on dung, carrion, fungi, vegetation, pollen, fruits, compost or roots. Unfortunately knowledge on the Indian scarab beetles is still poor. Only need of the hour is to utilize this potential boioresource in the interest of human society. The scarabs reported during this study are mainly phytophagous species (Brahmina sp., Holotrichia sp., Xylotrupes gideon, Anomala dimidiata, Anomala grandis). Apart from this, few coprophagous species (Catharsius molossus, Catharsius sagax and Onitis subopacus) are also recorded during this survey (Table 9). These beetles are dung feeders which feed on the dungs and excreta of the animals. Thus it helps in breaking down of plant and animal remains that contribute to the recycling of nutrients and the cleaning of the environment. The next dominant group of beetles in the tea ecosystems of North Bengal are Longhorn beetles or round-headed borers (Family Cerambycidae). Wood boring is carried out by various groups of insects either to obtain food or as means of protection of their eggs, larvae and pupae. Among the wood boring insects, the members of the family Cerambycidae are the most notorious pests of the freshly felled logs or dying standing trees. They mostly damage the shade trees of tea gardens and may also attack the tea plants. Majority of the cerambycid species are the borers of shade trees of tea gardens or other timber plants (Table 9). The members of the family Coccinellidae are mostly phytophagous but few species are also flower visitors and some are key predators that are conserved and augmented in agricultural ecosystems, to achieve biological control of pests. The other two families, Lucanidae are mostly rotten wood feeder and Curculionidae phytophagous (Table 9). Odonata are key organisms of the food web as predators both as larvae and as imagoes. They are among the dominant invertebrates predators in an ecosystem (Manwar NA et al., 2012). Present communication reports 12 species under 08 genera belonging to 02 families of the order Odonata from different tea gardens of North Bengal (Table 1). Worldwide orthopterans are considered of immense economic and ecological importance, because many species are accepted as pests of crops. Both nymphs and adults devour different kinds of vegetation, particularly succulent types. In ecology these insects provide food for the predators at all stages of life and some prey upon smaller invertebrates (Table 9).

Eventually it can be concluded that long term monitoring of insects and their taxonomic study in tea ecosystem is very much essential to understand their biological role (Pest, predator, pollinator and parasite) in the tea gardens of North Bengal. During this study, it has been observed that, apart from regular pests of tea 
plants there are many insects visited tea gardens those are recognised pests of other plants/crops. In future they may play a major role on the economy of tea gardens. Therefore, this study will definitely help to the tea gardens owner for making a full proof plan to control the pest and parasite attack in one side and in the other side conservation of the beneficial insects (Predator and pollinator) of the tea gardens.

\section{Acknowledgements}

Authors are grateful to Dr. Kailash Chandra, Director, Zoological Survey of India, Kolkata for providing necessary permissions to carry out this study and also for his constant support. Thanks are also due to Shri K. C. Gopi, Scientist ' $F$ ' for kindly going through the manuscript and making useful suggestions.

\section{References}

Ariyarathna, H.A.C.K., Arachchi, T.D.K. and Gunasekere, M.T.K. 2007. Floral Biology and Breeding System of Tea [Camellia sinensis (L.)]: Implications on the Tea Breeding Programme. S.L.J. Tea Sci., 72(2): 31-43.

Arora, G.S. 1986. Lepidoptera. In: Collection, Preservation and Identification of Insects and Mites of Economic Importance: 109-120. Published by the Director, Zoological Survey of India, Kolkata, India.

Bezbaruah, H.P. 1975. Development of flower, pollination and seed set in tea North-East India. Two and Bud, 22(1): 61-63.

Biswas, K.P. 2006. Description of tea plant. In: Encyclopaedia of Medicinal Plants: 964-966. Dominant Publishers and Distributors, New Delhi.

Biswas, O., Shah, S.K., Mishra, P., Mallick, K. and Mitra, B. 2015. Taxonomic Account of Erebidae (Lepidoptera: Noctuoidea) Pests of Tea gardens of West Bengal, India. Journal of Entomology and Zoology Studies, 3(5): 185-192.

Shanker, C., Mohan, M., Sampathkumar, M., Lydia, C. and Katti, G. 2013. Functional significance of Micraspis discolor (F.) (Coccinellidae: Coleoptera) in rice ecosystem. Journal of Applied Entomology. DOI: 10.1111/jen.12035.

Courtney, S.P., Hill, C.J. and Westerman, A. 1982. Pollen Carried for Long Periods by Butterflies. Oikos, 38: $260-263$.

Das, S., Roy, S. and Mukhopadhyay, A. 2010. Diversity of arthropod natural enemies in the tea plantations of North Bengal with emphasis on their association with tea pests. Current Science, 99(10): 1457-1463.

Dennis, D.S., and Lavigne, R.J. 2007. Hymenoptera as Prey of Robber Flies (Diptera: Asilidae) with New Prey Records. Journal of the Entomological Research Society, 9(3): 23-42.

Dennis, D.S., Lavigne, R.J. and Dennis, J.G. 2010. Hemiptera (Heteroptera/Homoptera) as Prey of Robber Flies (Diptera: Asilidae) with Unpublished Records. Journal of the Entomological Research Society, 12(1): 27-47.

Dennis, D.S., Lavigne, R.J. and Dennis, J.G. 2012. Spiders (Araneae) as Prey of Robber Flies (Diptera: Asilidae). J. Entomol. Res. Soc., 14(1): 65-76.

David, B.V., Ananthakrishnan, T.N. 2004. General and Applied Entomology (2 ${ }^{\text {nd }}$ Ed.). Tata McGraw-Hill Publishing Company Limited, New Delhi, pp. 391.

FAO. 2005. Committee on commodity problems: intergovernmental group on tea. Available at: http://www.fao. org/docrep/ meeting/009/j5602e.html

Faegri, K. and Van der Pijl, L. 1979. The Principles of Pollination Ecology. Pergamon, New York/Oxford, pp. 244.

Gurusubramanian, G., Rahman, A., Sarmah, M., Roy, S. and Bora, S. 2008. Pesticide usage pattern in tea ecosystem, their retrospects and alternative measures. J. Environ. Biol., 29(6): 813-26.

Hazarika, L.K., Bhuyan, M. and Hazarika, B.N. 2009. Insect pests of tea and their management. Ann. Rev. Entom., 54: $267-84$.

Jha, L.K. and Sen-Sharma, P.K. 2008. Forest Entomology. APH Pub. Corp., New Delhi, pp. 381.

Joern, A. and Rudd, N.T. 1982. Impact of predation by the robber fly Proctacanthus milbertii (Diptera: Asilidae) on grasshopper (Orthoptera: Acrididae) populations. Oecologia, 55(1): 42-46.

Khewa, S. and Mukhopadhyay, A. 2010. Biocontrol potential of a newly isolated bacterial agent against Arctornis submarginata (Walker) (Lepidoptera: Lymantriidae) occurring in Darjeeling Terai region. Journal of Biopesticides, 3(1 Special Issue): 114 -116.

Krenn, H.W., 2010. Feeding mechanisms of adult Lepidoptera: structure, function, and evolution of the mouthparts. Ann. Rev. Entom., 55: 307-327.

Kumar, A. 2002. Environmental Pollution and Agriculture. APR Publ. Corp., New Delhi, pp. 521.

Manwar, N.A., Rathod, P.P. and Ahmad Raja, I. 2012. Diversity and abundance of Dragonflies and Damselflies of Chatri Lake Region in Pohara - Malkhed Reserve Forest, Amravati, Maharashtra (India). International Journal of Engineering Research and Applications, 2(5): 521-523.

Mitra, B., Mishra, P. and Shah, S.K. 2014a. Orthoptera associated with the tea plants in North Bengal. Bionotes, $16(3)$ : 96.

Mitra, B., Shah, S.K., Mishra, P., Biswas, B., Hassan, M.E. and Saha, P.C. 2014b. Hemiptera associated with the tea plants in North Bengal. Bionotes, 16(4): 128-129. 
Mitra, B., Shah, S.K., Mishra, P., Mallick, K., Mazumder, A. and Bhattacharya, M. 2015. Butterfly Visitors in the Tea Gardens of North Bengal. Bionotes, 17(4): 92-93.

Mitra, B., Ghosh, J., Chakraborti, U., Shah, S.K. and Mishra, P. 2016. Beetle Fauna associated with the Tea Gardens of Northern West Bengal. Bionotes, 18(2): 66-67.

Mitra, B., Roy, S, Shah, S.K. and Mishra, P. 2017. Inventorisation of True flies (Insecta: Diptera) and their association with Tea plants in Dooars, West Bengal, India. International Journal of Entomology Research, 2(5): 21-26.

Nath, S., Rai, A., Chhetri, N. and Lepcha, P. 2009. Food preferences of a pest grasshopper, Atractomorpha

crenulata (Fabr.) (Orthoptera: Acrididae) from Darjeeling Hill. Entomon., 34(2): 111-113.

Roy, S., Das, S., Mukhopadhy, A. and Gurusubramanian, G. 2010. Bioefficacy of coccinellid predators on major tea pests. Jour. Biopestic. (Spl. Issue), 3(1): 33-36.

Ross, I.A. 2005. Tea common names and its uses: 1-19. In: Medicinal Plants of the World (3rd Vol.). Humana Press, New Jersey.

Roy, S., Muraleedharan, N. and Pujari, D. 2014. A catalogue of arthropod pests and their natural enemies in the tea ecosystem of India. Two and $a$ Bud, 61(1\&2): 11-39.

Sharma, G. 2011. Studies on Lepidopterous Insects Associated with Vegetables in Aravali Range, Rajasthan, India. Biological ForumAn International Journal, 3(1): 21-26.

Shah, S.K. and Mitra, B. 2015. 2Moth (Insecta: Lepidoptera) Fauna and their Insect Predators associated with the Tea gardens and the surrounding Natural Ecosystem Environs in Northern West Bengal, India .The Journal of Zoology Studies, (6): 1-5.

Shah, S.K., Mishra, P., Mondal, P., Dasgupta, S. and Mitra, B. 2015. Odonata associated with the Tea plantations in North Bengal. Bionotes, 17(2): 50.

Shelly, T.E. and Pearson, D.L. 1978. Size and Color Discrimination of the Robber Fly Efferia tricella (Diptera: Asilidae) as a Predator on Tiger Beetles (Coleoptera: Cicindelidae). DOI: http://dx.doi.org/10.1093/ee/7.6.790.

Stefanescu, C. and Traveset, A. 2009. Factors influencing the degree of generalization in flower use by Mediterranean butterflies. doi: 10.1111/j.1600-0706.2009.17274.x.

Shreeve, T.G. 1992. Adult behaviour: 22-45. In: Dennis, R.L.H. (Ed.). The ecology of butterflies in Britain, Oxford Univ. Press.

Tudor, O., et al. 2004. Flower preference of woodland butterflies in the UK: nectaring specialists are species of conservation concern. Biol. Cons., 403: 119: 397.

Wickramaratne, M.R.T., Vitarana, S.I. 1985. Insect pollination of tea (Camellia sinensis L.) in Sri Lanka. Tropical Agr., 623: 243-247

Wiklund, C., Eriksson, T. and Lundberg, H. 1979. The wood white butterfly Leptidea sinapis and its nectar plants: A case of mutualism or parasitism. Oikos, 33: 358-362.

Wiklund, C. 1981. On the pollination efficiency of butterflies: a reply to Courtney et al. Oikos, 38: 263. 